\title{
Some equinumerous pattern-avoiding classes of permutations
}

\author{
M. D. Atkinson \\ Department of Computer Science, University of Otago, New Zealand \\ received Feb 26, 2005, revised Mar 29, 2005, accepted Apr 27, 2005.
}

\begin{abstract}
Suppose that $p, q, r, s$ are non-negative integers with $m=p+q+r+s$. The class $X(p, q, r, s)$ of permutations that contain no pattern of the form $\alpha \beta \gamma$ where $|\alpha|=r,|\gamma|=s$ and $\beta$ is any arrangement of $\{1,2, \ldots, p\} \cup\{m-q+1, m-$ $q+2, \ldots, m\}$ is considered. A recurrence relation to enumerate the permutations of $X(p, q, r, s)$ is established. The method of proof also shows that $X(p, q, r, s)=X(p, q, 1,0) X(1,0, r, s)$ in the sense of permutational composition.
\end{abstract}

2000 MATHEMATICS SUBJECT CLASSIFICATION: 05A05

Keywords: permutation, pattern, enumeration

In their paper [2] Mansour and Vainshtein enumerated a three-parameter family of pattern-avoiding classes of permutations using fairly complex recurrence equations and generating functions. We shall derive a generalisation of their results by a very simple argument. Finally we observe that our proof gives a structure theorem for the pattern-avoiding classes in question.

To make this note self-contained we recall the notion of pattern-avoiding class. A permutation $\pi$ is said to be contained as a pattern in another permutation $\sigma$ if $\sigma$ has a subseqence order isomorphic to $\pi$ (its terms are ordered in the same relative way as $\pi$ ). If $\sigma$ does not contain $\pi$ it is said to avoid $\pi$. A pattern-avoiding class is a set of permutations defined by the property of avoiding some set of patterns. A central problem in the theory of pattern-avoiding classes is to enumerate them, i.e. to determine the number of permutations of each length in the class.

Let $p, q, r, s$ be non-negative integers and let $A(p, q, r, s)$ be the set of $(p+q) !(r+s)$ ! permutations of length $p+q+r+s$ which have the form $\alpha \beta \gamma$ where $|\alpha|=r,|\gamma|=s$ and $\beta$ is any arrangement of the $p$ smallest and $q$ largest values in the set $\{1,2, \ldots, p+q+r+s\}$.

Let $X(p, q, r, s)$ be the class of permutations that avoid all the permutations of this set. The following lemma gives a recursive characterisation of the permutations of this class and shows how they can be constructed.

Lemma 1 1. Let $\pi=\pi_{1} \cdots \pi_{n} \in X(p, q, r, s)$. Then there is at least one position $i$ of $\pi$ among the first $r$ or last $s$ positions whose value $\pi_{i}$ is among the $p$ smallest or $q$ largest values. If this term is removed from $\pi$ the resulting permutation $\pi^{\prime}$ (after appropriate renumbering) also lies in $X(p, q, r, s)$.

1365-8050 @ 2005 Discrete Mathematics and Theoretical Computer Science (DMTCS), Nancy, France 
2. Let $\pi^{\prime}=\pi_{1}^{\prime} \cdots \pi_{n-1}^{\prime} \in X(p, q, r, s)$. If we form a new permutation $\pi$ by inserting a new value $y$ (with appropriate renumbering of the old values) then, so long as we insert $y$ among the first $r$ or last s positions of $\pi^{\prime}$ and so long as $y$ is one of the $p$ smallest or q largest values in the new permutation, we have $\pi \in X(p, q, r, s)$.

Proof: To prove the first part, suppose no such position existed. Then all of the values $1,2, \ldots, p, n-q+$ $1, n-q+2, \ldots, n$ would occur among the positions $r+1, r+2, \ldots n-s+1$. Therefore the subsequence defined by the first $r$ and last $s$ positions together with the positions where the values $1,2, \ldots, p, n-q+$ $1, n-q+2, \ldots, n$ occur would be order isomorphic to a member of $A(p, q, r, s)$. Hence $\pi$ has such a position. If the term at this position was removed the resulting permutation would still avoid the patterns of $A(p, q, r, s)$.

The second part follows by observing that the insertion of $y$ into $\pi^{\prime}$ cannot create a subsequence order isomorphic to one in $A(p, q, r, s)$.

Theorem 2 Let $_{n}$ be the number of permutations of length $n$ that avoid all the permutations of $A(p, q, r, s)$. Let $u=p+q, v=r+s$. Then, for all $n \geq \max (u, v)$,

$$
t_{n}=u v t_{n-1}-2 !\left(\begin{array}{l}
u \\
2
\end{array}\right)\left(\begin{array}{l}
v \\
2
\end{array}\right) t_{n-2}+3 !\left(\begin{array}{l}
u \\
3
\end{array}\right)\left(\begin{array}{l}
v \\
3
\end{array}\right) t_{n-3}-4 !\left(\begin{array}{l}
u \\
4
\end{array}\right)\left(\begin{array}{l}
v \\
4
\end{array}\right) t_{n-4}+\cdots
$$

Proof: Consider permutations of length $n$ that avoid all the permutations of $A(p, q, r, s)$. We shall call the $p$ smallest and $q$ largest values the critical values of the permutation and the $r$ initial and $s$ final positions the critical positions. Furthermore, we call a critical value that occurs in a critical position a critical term. Lemma 1 shows that the permutations of $X(p, q, r, s)$ are precisely those with at least one critical term which, if removed, results in a permutation of $X(p, q, r, s)$.

Since there are $u$ critical values and $v$ critical positions there are $\left(\begin{array}{l}u \\ k\end{array}\right)\left(\begin{array}{l}v \\ k\end{array}\right) t_{n-k}$ permutations with exactly $k$ critical terms which, if removed, result in a permutation of $X(p, q, r, s)$. Therefore, by inclusionexclusion, there are

$$
u v t_{n-1}-2 !\left(\begin{array}{l}
u \\
2
\end{array}\right)\left(\begin{array}{l}
v \\
2
\end{array}\right) t_{n-2}+3 !\left(\begin{array}{l}
u \\
3
\end{array}\right)\left(\begin{array}{l}
v \\
3
\end{array}\right) t_{n-3}-4 !\left(\begin{array}{l}
u \\
4
\end{array}\right)\left(\begin{array}{l}
v \\
4
\end{array}\right) t_{n-4}+\cdots
$$

permutations with at least one such critical term.

The recurrence relation given in this theorem has $\lambda=\min (u, v)$ terms on the right hand side and is valid for all $n \geq \mu=\max (u, v)$. We may apply the standard method for deriving the ordinary generating function $\sum t_{n} x^{n}$; that is to say, we multiply the recurrence by $x^{n}$ and sum over all $n \geq \mu$. Such a calculation expresses the generating function as a rational function in the form

$$
\sum t_{n} x^{n}=\frac{p(x)}{q(x)}
$$

where the denominator is directly obtained from the recurrence as

$$
q(x)=1-u v x+2 !\left(\begin{array}{l}
u \\
2
\end{array}\right)\left(\begin{array}{l}
v \\
2
\end{array}\right) x^{2}-3 !\left(\begin{array}{l}
u \\
3
\end{array}\right)\left(\begin{array}{l}
v \\
3
\end{array}\right) x^{3}+4 !\left(\begin{array}{l}
u \\
4
\end{array}\right)\left(\begin{array}{l}
v \\
4
\end{array}\right) x^{4}+\cdots
$$


and has degree $\lambda$. The numerator can be ascertained from the initial terms of the sequence $\left(t_{n}\right)$ which, for $n<u+v$, are equal to $n$ !. After some algebraic manipulation we obtain

$$
p(x)=\sum_{i=0}^{\lambda-1} x^{i} i ! \sum_{j=0}^{i}(-1)^{j} \frac{\left(\begin{array}{l}
u \\
j
\end{array}\right)\left(\begin{array}{l}
v \\
j
\end{array}\right)}{\left(\begin{array}{l}
i \\
j
\end{array}\right)}+(-1)^{\lambda} x^{\lambda} \lambda ! \sum_{i=0}^{\mu-\lambda-1} x^{i} i !\left(\begin{array}{c}
\mu-i-1 \\
\lambda
\end{array}\right) .
$$

This generating function is the same as the one given in the main theorem of [2] for the enumeration of another pattern-avoiding set of permutations. This is no coincidence since, by putting $q=0$, we can obtain that theorem as follows. The permutations of $A(p, 0, r, s)$ are precisely those where the values $1,2, \ldots, p$ occur (in any order) at positions $r+1, r+2, \ldots, r+p$. The inverses of these permutations are those where positions $1,2, \ldots, p$ contain the values $r+1, r+2, \ldots, r+p$ (in any order); this is exactly the set of avoided permutations considered in [2] (see the discussion following Lemma 2.1 of that paper). However, permutation inversion is a symmetry of the pattern-avoidance relation and so the classes $X(p, 0, r, s)$ have the same enumerations as the classes in [2].

Finally we give a structural property of $X(p, q, r, s)$. Lemma 1 shows that every permutation $\pi \in$ $X(p, q, r, s)$ can be generated as the output of a certain process that has the sequence $1,2, \ldots, n$ as input. This process has $n$ steps in each of which one value from the remaining input is moved to a position of the output permutation. The input value moved is chosen from among the $p$ smallest and $q$ greatest values remaining. The position where it is placed is chosen from among the first $r$ and last $s$ positions still undefined.

This process can be regarded as taking place in two stages. In the first stage the sequence of values is chosen and placed, from left to right, in a permutation $\gamma$. In the second stage the terms of $\gamma$ are processed sequentially and inserted according to how the output positions are chosen. The permutation $\gamma$ generated by the first stage lies in $X(p, q, 1,0)$ while the permutation corresponding to the second stage lies in $X(1,0, r, s)$. It follows that

$$
X(p, q, r, s)=X(p, q, 1,0) X(1,0, r, s)
$$

in the sense of composition of permutations (see [1] for an account of the composition operation on pattern-avoiding classes).

\section{References}

[1] M. H. Albert, R. E. L. Aldred, M. D. Atkinson, H. P. van Ditmarsch, C. C. Handley, D. A. Holton, D. J. McCaughan: Compositions of pattern restricted sets of permutations, Technical Report OUCS2004-12, Department of Computer Science, University of Otago, 2004.

[2] T. Mansour, A. Vainshtein: Avoiding maximal parabolic subgroups of $S_{k}$, Discrete Mathematics and Theoretical Computer Science 4 (2000), 67-77. 
